\title{
TRANSTORNOS FÓBICO-ANSIOSOS: ABORDAGEM EPIDEMIOLÓGICA DAS INTERNACOES HOSPITALARES
}

Camila Câmara Pereira da Paz ${ }^{1}$, Isabela Luiza Moreira Brant¹ ${ }^{1}$, Mayara Sanches Marques', Richardson Miranda Machado²

\begin{abstract}
RESUMO: Trata-se de um estudo descritivo e retrospectivo realizado no banco de dados do Sistema de Internações Hospitalares e Prontuários, dos anos de 1980 a 2005, de uma clínica psiquiátrica de Divinópolis - Minas Gerais com o objetivo de caracterizar os pacientes hospitalizados acometidos por transtornos fóbico-ansiosos. Entre 24.161 admissões no período, 1.113 (4,6\%) pacientes foram acometidos por transtornos fóbico-ansiosos; sendo 675 (60,7\%) homens; 298 (26,8\%) na faixa etária de 10 a 20 anos; 518 (46,54\%) procedentes de Centros de Atenção Psicossocial; 517 (53,6\%) permaneceram internados por até 30 dias; 976 (87,7\%) constituíram primeira internação; 677 (60,9\%) estavam internados pelo Sistema Único de Saúde e 1.010 (90,8\%) tiveram alta médica. A detecção e tratamento precoces podem contribuir para a desospitalização e abordagem do paciente em seu contexto social.

DESCRITORES: Transtornos de ansiedade; Transtornos fóbicos; Enfermagem; Saúde mental.

\section{PHOBIC ANXIETY DISORDERS: AN EPIDEMIOLOGICAL APPROACH TO HOSPITAL INPATIENT TREATMENT}

ABSTRACT: This is a descriptive and retrospective study, undertaken with the database of the Hospitalisation and Health Records System, regarding the years 1980 to 2005, in a psychiatric clinic in Divinópolis in the state of Minas Gerais, with the objective of characterizing the hospitalized patients who had been affected by phobic anxiety disorders. Of the 24,161 admissions in the period, $1,113(4.6 \%)$ patients had been affected by phobic anxiety disorders; of whom 675 (60.7\%) were male; 298 (26.8\%) in the age range of 10 to 20 years of age; 518 (46.54\%) came from Psycho-Social Care Centers; 517 (53.6\%) remained hospitalized for up to 30 days; 976 (87.7\%) were being hospitalized for the first time; 677 (60.9\%) were hospitalized under the Unified Health System and 1,010 (90.8\%) were medically discharged. Early detection and treatment can contribute to de-hospitalization and treating the patient in his or her social context.

DESCRIPTORS: Anxiety disorders; Phobic disorders; Nursing; Mental health.

\section{TRASTORNOS FÓBICO ANSIOSOS: ABORDAJE EPIDEMIOLÓGICO DE LAS INTERNACIONES HOSPITALARES}

RESUMEN: Este es un estudio descriptivo y retrospectivo realizado en el banco de datos del Sistema de Internaciones Hospitalares y Prontuarios, de los años de 1980 a 2005, de una clínica psiquiátrica de Divinópolis - Minas Gerais, con el objetivo de caracterizar los pacientes hospitalizados víctimas de trastornos fóbico y ansiosos. Entre 24.161 admisiones en el periodo, 1.113 (4,6\%) pacientes fueron víctimas de trastornos fóbico ansiosos; siendo 675 (60,7\%) hombres; 298 $(26,8 \%)$ en la franja etaria de 10 a 20 años; 518 (46,54\%) procedentes de Centros de Atención Psicosocial; 517 (53,6\%) permanecieron internados hasta 30 días; 976 (87,7\%) constituyeron primera internación; 677 (60,9\%) estaban internados por el Sistema Único de Salud y 1.010 (90,8\%) tuvieron alta médica. La detección y tratamiento precoces pueden contribuir para la "desospitalización" y abordaje del paciente en su contexto social.

DESCRIPTORES: Trastornos de ansiedad; Trastornos fóbicos; Enfermería; Salud mental.

*Artigo desenvolvido durante a disciplina optativa Pesquisa em Saúde' do Curso de Graduação em Enfermagem da Universidade Federal de São João Del Rei - UFSJ, Campus Centro Oeste Dona Lindu - CCO.

${ }^{1}$ Acadêmica do Curso de Enfermagem da UFSJ CCO.

${ }^{2}$ Enfermeiro. Doutor em Psiquiatria. Professor de Psiquiatria e Coordenador da Disciplina Optativa Pesquisa em Saúde da UFSJ CCO.

Autor correspondente:

Camila Câmara Pereira da Paz

Universidade Federal de São João Del Rei

R. Dr. Pedro Ruela, 180 - 31570-100 - Belo Horizonte-MG-Brasil

E-mail: camila_paz@live.com
Recebido: 02/07/2012

Aprovado: 04/12/2012

Cogitare Enferm. 2013 Jan/Mar; 18(1):136-41 


\section{INTRODUÇÃO}

Os transtornos fóbico-ansiosos são definidos como um grupo de distúrbios psíquicos nos quais uma ansiedade é desencadeada exclusiva ou essencialmente por situações nitidamente determinadas que não apresentam perigo real. Estas situações são, por esse motivo, evitadas ou suportadas com temor ${ }^{(1)}$. Esses transtornos podem ser denominados como transtornos de ansiedade (TA) e são subdivididos segundo o Manual Diagnóstico e Estatístico de Transtornos Mentais ${ }^{(2)}$ em categorias, dentre as quais destacam-se: ataque de pânico; transtorno de pânico com ou sem agorafobia; fobias específicas e fobia social; transtorno de ansiedade generalizada e transtorno de estresse pós-traumático. $\mathrm{O}$ transtorno de ansiedade de separação é o único definido como exclusivo da infância e adolescência.

A ansiedade fóbica pode estar associada a uma depressão. Portanto, para determinar se convém fazer dois diagnósticos (ansiedade fóbica e episódio depressivo) ou um só (ansiedade fóbica ou episódio depressivo) é importante que o profissional leve em conta a ordem de ocorrência dos transtornos e as medidas terapêuticas que são consideradas no momento do exame ${ }^{(1)}$.

Pesquisadores $^{(3)}$ reforçam a ideia de que os transtornos ansiosos estejam entre os mais prevalentes e potencialmente mais incapacitantes do ponto de vista da saúde pública. Em pesquisa envolvendo as cidades de Porto Alegre, São Paulo e Brasília foram avaliados 6.470 indivíduos, dessa amostra os transtornos ansiosos foram tidos como sendo os mais prevalentes $(17,6 \%)$, seguidos pelos transtornos fóbicos (16,7\%).

Cabe ressaltar que a ansiedade é tida como uma manifestação normal do nosso organismo frente a situações especiais. Tal ansiedade fisiológica nos deixa em alerta para a detecção de ameaças futuras além de modular nosso funcionamento cognitivo. Já os TA são diagnosticados quando os quadros ansiosos estão presentes de forma extrema, duradoura e são desproporcionais à situação. Esses quadros são caracterizados por manifestações sistêmicas (sudorese, taquicardia, boca seca, medo, insônia, por exemplo) e cognitivas (apreensão, nervosismo, irritabilidade e desatenção) ${ }^{(3)}$.

Os TA quando presentes na infância ou na adolescência têm grande possibilidade de se agravar ao longo da $v i^{(2)}$ o que, além de trazer consequente prejuízo funcional imediato, leva a possíveis implicações de médio e longo prazo na vida do indivíduo, como a diminuição da autoestima e o desinteresse de viver.
Diante dessas considerações temos como propósito traçar o perfil epidemiológico dos indivíduos portadores dos transtornos fóbico-ansiosos na região Centro-Oeste de Minas Gerais e caracterizá-los quanto ao sexo, idade, procedência para a internação, tempo de internação, tipo de alta hospitalar e fonte financiadora da internação. Deste modo ampliaremos a disponibilidade de informações essenciais para o estabelecimento de medidas que melhor atendam as necessidades dos indivíduos portadores de tal doença.

\section{MÉTODO}

Trata-se de um estudo descritivo e retrospectivo, realizado em uma clínica psiquiátrica sediada em Divinópolis/Minas Gerais. Essa clínica é considerada centro de referência do Sistema Único de Saúde (SUS) para as internações de indivíduos com transtornos psiquiátricos agudos ou crônicos na região Centro-Oeste de Minas Gerais.

A clínica tem a finalidade de reinserir o portador de transtorno mental no meio social, assim como, capacitar a família para melhor receber este indivíduo; oferece assistência de caráter ambulatorial, de urgência e internação aos indivíduos de ambos os sexos residentes na região ou em cidades adjacentes. A instituição possui 120 leitos, sendo 85\% destinados ao SUS e $15 \%$ ao sistema particular de atendimento $^{(4)}$. A clínica acompanha e armazena as informações das internações hospitalares por meio do seu Sistema de Internação Hospitalar (SIH) e de prontuários arquivados no Setor de Arquivo Médico e Estatístico (SAME). A coleta de informações foi realizada através do SIH e SAME da instituição.

As variáveis utilizadas para o estudo foram sexo; idade; procedência do paciente; tempo de internação; tipo de alta hospitalar; tipo de internação e fonte financiadora da internação. Foram adotados como critérios de inclusão: 1) paciente internados na clínica no período de 20 de outubro de 1980 (data de inauguração da clínica) a 31 de dezembro de 2005; 2) com permanência superior a 24 horas; 3) acompanhados desde a admissão, alta ou transferência. Os dados foram coletados após aprovação do Comitê de Ética em Pesquisa da Universidade de São Paulo/ Campus de Ribeirão Preto, sob parecer n. 0649/ USP/RP. A análise dos dados foi realizada através do Software Statiscal Package for Social Sciences (SPSS) versão 11.5 e os resultados apresentados na forma de tabelas. 


\section{RESULTADOS}

No período estudado, dos 24.161 indivíduos admitidos na clínica psiquiátrica, 1113 foram acometidos por transtornos fóbico-ansiosos, o que representa uma taxa de $4,6 \%$. A tabela 1 apresenta as características sociodemográficas e a tabela 2 as características da internação destes pacientes.

Tabela 1- Caracterização sociodemográfica dos pacientes admitidos na clínica psiquiátrica devido a transtornos fóbico-ansiosos de 1980 a 2005. Divinópolis, 2011

\begin{tabular}{llcc}
\hline Variáveis & Características \\
& Sociodemográficas & n & \% \\
\hline \multirow{2}{*}{ Sexo } & Masculino & 676 & 60,7 \\
& Feminino & 437 & 39,3 \\
\hline \multirow{5}{*}{ Idade } & 10 a 20 anos & 299 & 26,8 \\
& 21 a 30 anos & 287 & 25,7 \\
& 31 a 40 anos & 163 & 14,6 \\
& 41 a 50 anos & 295 & 26,5 \\
& 51 a 60 anos & 62 & 5,5 \\
& 61 a 70 anos & 06 & 0,5 \\
& $>70$ anos & 01 & 0,08 \\
\hline \multirow{4}{*}{ Procedência } & Consultório Médico & 435 & 39,0 \\
& Particular & & \\
& Pentros de Atenção & 518 & 46,5 \\
& Família & 98 & 8,8 \\
& Outros & 62 & 5,5 \\
\hline Fonte: Banco de dados do SIH/CSBM & &
\end{tabular}

Tabela 2 - Características clínicas dos pacientes admitidos na clínica psiquiátrica devido a transtornos fóbico-ansiosos de 1980 a 2005. Divinópolis, 2011

\begin{tabular}{llcc}
\hline Variáveis & Características & n & \% \\
\hline \multirow{3}{*}{ Clínicas } & 1 a 30 dias & 597 & 53,6 \\
ternação de & 31 a 60 dias & 413 & 37,1 \\
& 61 a 90 dias & 57 & 5,1 \\
& > 90 dias & 46 & 4,1 \\
\hline \multirow{4}{*}{ Tipo de Alta } & Alta Médica Hospitalar & 1011 & 90,8 \\
Hospitalar & Alta a Pedido & 38 & 3,4 \\
& Alta Administrativa & 01 & 0,08 \\
& Transferência Clínica & 27 & 3,2 \\
\hline Tipo de & Primeira internação & 978 & 87,8 \\
Internação & Reinternação & 135 & 12,2 \\
\hline Fonte & Sistema Unico de Saúde & 678 & 60,9 \\
Financiadora & Particular & 136 & 12,2 \\
da Internação & Convênios & 299 & 26,8 \\
\hline
\end{tabular}

Fonte: Banco de dados do SIH/CSBM

\section{DISCUSSÃO}

Observamos maior prevalência dos TA no sexo masculino (676-60,7\%), o que contraria os resultados de outros estudos, os quais demonstraram maior ocorrência nas mulheres e consideraram como uma das possíveis causas a constante flutuação dos hormônios sexuais femininos, como o estrogênio, que agem na modulação do humor, desde a menarca até a menopau$\mathrm{sa}^{(5-6)}$. Em nossa compreensão essa divergência se dá pelo fato de que os homens, na maioria das vezes, são mais resistentes à procura prematura de assistência a saúde, o que poderia iniciar-se, por exemplo, na atenção primária de saúde. Deste modo, quando procuram os serviços de saúde já apresentam quadros patológicos mais graves, os quais requerem tratamento intensivo, resultando assim no grande número de internações hospitalares.

Autores $^{(6)}$ afirmam que a baixa adesão pelos homens aos cuidados de saúde pode ser ocasionada tanto pela resistência cultural do sexo masculino em frequentar regularmente os serviços de saúde quanto pela deficiência desses serviços em acolher essa população. Assim, comprometendo a avaliação e o tratamento de diversas morbidades no gênero masculino.

As fobias, em geral, iniciam-se na infância ou na adolescência, exceto aquelas de origem traumática, as quais se iniciam em qualquer período posterior ao trauma ${ }^{(7)}$. Dados epidemiológicos sugerem que os TA tenham maior prevalência entre adolescentes do que em crianças e indivíduos adultos. Estes inquéritos ainda afirmam que os casos que se iniciam na adolescência têm maior risco de se estender até a idade adulta, e raramente apresentam remissão ${ }^{(7-8)}$.

Os dados encontrados neste estudo corroboram com os registros apontados por outros estudos ${ }^{(7-8)}$, ou seja, os TA foram mais prevalentes entre adolescentes $(299-26,6 \%)$. Pesquisadores ${ }^{(6,9)}$ ressaltam que na maioria dos casos o desenvolvimento de TA pelos jovens está associado aos instintos naturais de um ser em fase peculiar de desenvolvimento, com a vontade de se tornar independente, a opressão social ou econômica, ou até mesmo por perturbações hormonais.

No que se refere a faixa etária acometida por TA verificamos que a medida com que se envelhece a prevalência de TA diminui. Ou seja, entre quem não desencadeou o transtorno durante a infância ou adolescência a chance de desenvolvê-lo na terceira idade cai consideravelmente. Essa redução se deve, talvez, pelo fato de que com o avançar da idade os TA podem

Cogitare Enferm. 2013 Jan/Mar; 18(1):136-41 
ser mascarados por outros quadros patológicos, tais como demências e depressão( ${ }^{(9)}$. Assim como, pode estar relacionado com menor produção e/ou flutuação hormonal, o que proporciona menores alterações no humor e, consequentemente, um menor acometimento pelas fobias ansiosas.

Outro fator que pode estar relacionado ao decréscimo da prevalência de TA com o avançar da idade, pode ser a redução na procura pelos serviços de saúde mental pelos idosos. O que pode ser explicado pela dificuldade de mobilidade, crenças e atitudes dos idosos frente aos transtornos mentais, incapacidade dos idosos de perceber a ocorrência de transtornos mentais e interpretação das manifestações como características naturais do processo de envelhecimento ${ }^{(10)}$.

Ao analisarmos a procedência dos pacientes acometidos por TA percebermos que o Centro de Atenção Psicossocial (CAPS) foi o responsável pelo maior número de encaminhamentos para a internação hospitalar. Esses dados vão contra o propósito da Reforma Psiquiátrica, que visa manejar o paciente psiquiátrico em nível extra-hospitalar a fim de evitar a permanência desnecessária destes por longos períodos em hospitais psiquiátricos e prevenir sua segregação social ${ }^{(11)}$.

Estudos $^{(6,10)}$ demonstram a falta de investimentos nos CAPS já existentes, a falta de abertura de novos CAPS, bem como de novos serviços extra-hospitalares para o tratamento dos transtornos mentais. Isso resulta na manutenção do sistema hospitalar para o tratamento dos transtornos psiquiátricos, pelo próprio SUS, contrariando a Lei 10.216 da Reforma Psiquiátrica ${ }^{(11)}$.

No que se refere ao tempo de internação pós TA, verificamos que a maioria das internações teve duração de até 30 dias; supomos tratar-se de casos agudos, e nos quias sinais e sintomas dos transtornos se deram pela primeira vez. Assim, os sintomas desaparecem com mais rapidez e o individuo não apresenta risco para si próprio e para os demais, permitindo a alta hospitalar dentro de um curto período de tempo ${ }^{(12)}$. Já os casos de internação entre 31 a 60 dias podem estar relacionados com casos considerados crônicos, ou em fase de transição agudo-crônico, na qual o indivíduo oferece risco para sua integridade física e a de outros ${ }^{(8)}$.

O grande número de pacientes com alta hospitalar por consentimento médico representa boa adesão ao tratamento proposto e reflete uma oportunidade de se alcançar adequada estabilidade psicoterapêutica e farmacoterápica dos indivíduos com TA. Alguns autores ${ }^{(4,9,1)}$ ressaltam a importância da promoção de determinados fenômenos multifatoriais tidos como responsáveis pela adesão ao tratamento pelo paciente com TA. Dentre estes fenômenos está a motivação do paciente para o tratamento/melhora, a interpretação positiva em relação aos resultados do tratamento, o reconhecimento do transtorno fóbico como uma doença, assim como, o estabelecimento de relacionamento interpessoal positivo com a equipe de saúde e a presença de suporte familiar.

Quanto à classificação das internações, foram majoritariamente registradas como primeira internação. $\mathrm{O}$ número reduzido de reinternações pode ser explicado como resultado do movimento da Reforma Psiquiátrica, cuja Lei 10.216 (06 de abril de 2001) dispõe sobre a proteção e os direitos das pessoas portadoras de transtornos mentais e direciona o modelo assistencial em saúde mental. Ainda, promove e cobra que os pacientes portadores de sofrimento psíquico sejam tratados em serviços extra-hospitalares, ou seja, em Ambulatórios, Núcleos de Atenção Psicossocial (NAPS), Centros de Atenção Psicossocial (CAPS), Oficinas Terapêuticas, dentre outros. Deste modo, promovendo novas formas de cuidar em Saúde Mental e buscando uma nova prática inserida na sociedade, ou seja, no contexto social dos pacientes, no qual se eclode e desencadeia todo o processo de adoecimento ${ }^{(11)}$.

Ao analisarmos a fonte financiadora da internação foi possível remeter a um dos princípios doutrinários dos SUS - a Universalidade, tendo a saúde como direito de todos e dever do Estado. O SUS foi o responsável pelo financiamento da maioria das internações por TA. Porém, devemos ressaltar que o SUS ainda investe muito no nível terciário de atenção a saúde, muitas vezes negligenciando a importância do trabalho assistencial que a atenção primária exerce ${ }^{(12)}$. É através da Estratégia de Saúde da Família (ESF) que o paciente com transtorno psiquiátrico tem o contato inicial com o serviço de saúde. Sendo assim, tal nível de atenção, quando bem organizado e composto por profissionais engajados na busca ativa e na assistência integral, tem a capacidade de realizar intervenções sem precisar encaminhá-los para os níveis mais complexos de assistência. Evitando assim, a sobrecarga no atendimento, internações desnecessárias e os altos gastos com a assistência a saúde ${ }^{(10)}$.

A promoção da saúde mental e a luta pela desospitalização passam pelo efetivo financiamento e implantação, pelo SUS, de uma rede comunitária que previna as situações de crise e ofereça alternativas à internação hospitalar. Para isso, há que se aumentar a oferta e a eficácia dos atendimentos dos Centros de Atenção Psicossocial e promover o desenvolvimento de ações conjuntas 
com a ESF, de acordo com o grau de complexidade que a situação exige, no atendimento às reais necessidades dos portadores de sofrimento psíquico, dentre eles os acometidos por TA e suas famílias.

Assim, a inserção e racionalização dos serviços de saúde mental na atenção básica, por intermédio da Estratégia de Saúde da Família (ESF), torna-se oportuna, pois cria a possibilidade de estender a cobertura em saúde mental a maior número de usuários. Além de promover o atendimento e o acompanhamento dos pacientes portadores de TA em seu próprio cenário de vida, sem desvinculá-lo do seu contexto social e prevenindo a sua segregação.

\section{CONSIDERAÇÕES FINAIS}

Observamos que os homens apresentaram um número maior de casos de TA, o que contraria a maioria dos estudos que demonstram que esse transtorno é mais frequente em mulheres. Isso é algo que deve ser investigado para que se possam ter níveis reais da ocorrência de TA nos dois sexos, e assim elaborar políticas de saúde de acordo com a necessidade de cada gênero.

Vimos que os TA acometem com mais frequência adolescentes, mostrando a necessidade de se ter mais atenção ao observar indivíduos nessa faixa etária ao se considerar que o tratamento precoce permite maiores chances de se alcançar a cura ou a remissão da doença. Neste sentido, torna-se essencial a inserção e racionalização dos serviços de saúde mental na atenção primária de saúde, por intermédio da ESF. Proporciona-se, deste modo, a possibilidade de estender a cobertura em Saúde Mental a um maior número de usuários, além de promover o atendimento e o acompanhamento precoce dos pacientes portadores de transtornos mentais menos graves em seu próprio cenário de vida, sem desvinculá-los do seu contexto social.

Deste modo, a Enfermagem em Saúde Mental, profissão de expressiva importância e atuação, passa a ser percebida como recurso primordial para a promoção da saúde mental dos usuários, exigindo novos modos de tratar e de acolher pessoas em sofrimento, principalmente o portador de TA, na sua vida diária e em seu espaço comunitário.

O TA é uma patologia cada vez mais frequente em nossa sociedade e que carece de maior divulgação de informações sobre a doença para a população, e para os profissionais de saúde, principalmente no que se referem aos sinais, sintomas e a necessidade do diagnóstico e tratamento precoces. É importante ressaltar que as informações acerca do diagnóstico, etiologia e características principais da doença devem ser continuamente revistas e atualizadas pelos profissionais da saúde, pois tornam os diagnósticos e a propedêutica mais eficazes, reduzindo o estigma social gerado pela doença e o impacto econômico para o sistema de saúde.

É evidente a importância da avaliação do paciente com TA por uma equipe multidisciplinar, bem como, a promoção do tratamento. Esse cuidado contribui para uma recuperação mais rápida do paciente e a prevenção de reincidência do quadro patológico. Assim, diminuindo o número e o tempo das internações, bem como os gastos com o tratamento e o afastamento dos pacientes das suas atividades.

No estudo foi perceptível a manutenção do sistema hospitalocêntrico para o tratamento psiquiátrico, seja pelo SUS por meio do financiamento dos leitos da instituição hospitalar, seja pelos consultórios particulares e pelos CAPS, que emitem encaminhamentos para a internação. Neste sentido, verificamos a necessidade de se fazer valer a Lei 10.216 da Reforma Psiquiátrica, por meio de investimentos na consolidação do CAPS como referência para a assistência em saúde mental. E, de forma, a constituir junto a outros dispositivos assistenciais (Equipes de Saúde da Família; Residências Terapêuticas; leitos em hospitais gerais; ambulatórios), uma rede de cuidados em saúde mental. Esta rede funcionando de forma articulada, tendo o CAPS como referência na organização, regulação e na promoção da atenção psicossocial aos pacientes com transtornos mentais.

\section{REFERÊNCIAS}

1. Centro Colaborador da OMS para a Classificação de Doenças em Português - CBCD. Classificação Estatística Internacional de Doenças e Problemas Relacionados à Saúde - CID-10. Brasília, DF: Ministério da Saúde; 2011.

2. Vianna RRAB, Campos AA, Fernandez JL. Transtornos de ansiedade na infância e adolescência: uma revisão. Rev. bras. ter. cogn. 2009;5(1):73-9.

3. Ramos RT. Transtornos de ansiedade: a prática clínica em doenças reumáticas. Rev Bras Med. 2009;66(11):9-14.

4. Irmãs Hospitaleiras. Clínica São Bento Menni. Divinópolis, MG. [acesso em 29 set 2011]. Disponível: http://www. hospitaleiras.org/index.php?irmas=Nav/Clinica.

5. De Andrade LHSG, Viana MC, Silveira CM. Epidemiologia dos transtornos psiquiátricos na mulher. Rev. psiquiatr. clín. 2006;33(2):43-54.

Cogitare Enferm. 2013 Jan/Mar; 18(1):136-41 
6. Couto MT, Pinheiro TF, Valença O, Machin R, Da Silva GSN, Gomes R, et al. O homem na atenção primária à saúde: discutindo (in)visibilidade a partir da perspectiva de gênero. Interface- Comunic., Saude, Educ. 2010;33(14):257-70.

7. Terra MB, Garcez JP, Noll B. Fobia específica: um estudo transversal com 103 pacientes tratados em ambulatório. Rev. psiquiatr. clín. 2007;34(2):68-73.

8. Isolan L, Pheula G, Manfro GG. Tratamento do transtorno de ansiedade social em crianças e adolescentes. Rev. psiquiatr. clín. 2007;34(3):125-32.

9. Clemente AS, Filho AIL, Firmo JOA. Concepções sobre transtornos mentais e seu tratamento entre idosos atendidos em um serviço público de saúde mental. Cad. Saúde Pública. 2011;27(3):555-64.

10. Barros REM, Tung TC, Mari JJ. Serviços de emergência psiquiátrica e suas relações com a rede de saúde mental brasileira. Rev. Bras. Psiquiatr. 2010;32(Suppl II):94-106.

11. Kilsztajn S, Lopez ES, Lima LZ, Rocha PAF, Carmo MSN. Leitos hospitalares e reforma psiquiátrica no Brasil. Cad. Saúde Pública. 2008;24(10):2354-62.

12. Porto SM, Santos IS, Ugá MAD. A utilização de serviços de saúde por sistema de financiamento. Ciênc. saúde colet. 2006;11(4):895-910. 